\title{
Study on the Morphological Development of Quail Embryos
}

http://dx.doi.org/10.1590/1806-9061-2015-0177

Technical Note

\section{-Author(s)}

\section{Bai JYI \\ Pang $Y Z^{\prime}$ \\ Zhang $\mathrm{XH}^{\prime}$}

Li YX'

College of Animal Science and Technology, Henan University of Science and Technology - No. 70 Tian Jin Road Jian Xi District, Luoyang, Hennan province 471003, People' $s$ Republic of China, Henan Luoyang 471003 China

\section{Mail Address}

Corresponding author e-mail address Jun Yan Bai

College of Animal Science and Technology, Henan University of Science and Technology, Kaiyuan Road in Luolong District of Henan city of Luoyang Province, No. 263, China

Phone: +86-13721673010

Email: junyanbai@163.com

\section{- Keywords}

Quail, Morphological observation, Embryo length, Regression equation.

\section{ABSTRACT}

In this study, the SWKQ series microcomputer automatic incubator was used to study the growth and development of quail in the embryonic stage. Results showed that the embryo shape of became gradually defined as embryo aged. On day 6 , the head and body of quail were clearly differentiated, the legs became longer and the wings appeared. At 7 embryo age, the entire embryo of quail was very clear, and the beak has formed. During 3 to 9 days of age, quail embryos length increased quickly, showing a linearly upward trend. At 9 day old, quail embryos length reached $2.2 \mathrm{~cm}$. The regression equation of embryo length to day old was curve regression, giving as following: $y=-0.464+0.325 x-0.004 x^{2}, y$ : embryo length, $x$ : the age of the embryo.

\section{INTRODUCTION}

Quails have some characteristics, such as small size, short generation interval, that makes them an ideal candidate as experimental animal models for nutrition, genetics, epidemiology, histology, embryology, physiology, reproductive physiology, and pharmacology research (Pang, 2009). The current domestic quails originate from the wild Japanese quail (Coturnix japonica), and it is mainly divided into commercial and experimental types. Commercial quails can be further divided into eggtype and meat-type quails, according to its economic use. Egg-type quails include mostly Japanese quails, North Korean quails, Beijing white-feather quails, Yellow Feather quails, Estonian quails, as well as and those autosexing lines, composed of white, yellow, and Korean Longcheng lines. Meat-type breeds include the French FM and BC genetic strains, Chinese White Feather quails, and Ssha Vee Mai To quails (Chang, 2003; Lin, 2000).

The hatching rate of quails directly influences the incubation conditions, the health of the hatchlings, and their production performance in the field. Several methods are applied for the artificial incubation of quails, including heated floor, heated barrels, electric incubator, and mechanical incubator (Lin, 2000; Chang, 2003). However, there are few reports on the growth and development of quail embryos under artificial incubation conditions. Therefore, the objective of this experiment was to study the growth and development of Beijing White Feather quail embryos and causes of embryo mortality.

\section{MATERIALS AND METHODS}

\section{Eggs}

One hundred Beijing White Feather quail eggs laid by a flock of quails reared on pasture in the farm experimental of Henan University of Science and Technology, China, were used. 


\section{Incubation}

Eggs were set in an incubator (SWKQ-8D, Three Giant Technology Co., Ltd. Hefei, China) with full automatic control of a microcomputer was utilized for the constant temperature incubation (Figure 1). The egg surface was sprayed with potassium permanganate solution (1 $\mathrm{g} \mathrm{KMnO} 4: 500 \mathrm{ml} \mathrm{H}_{2} \mathrm{O}$ ) for disinfection.

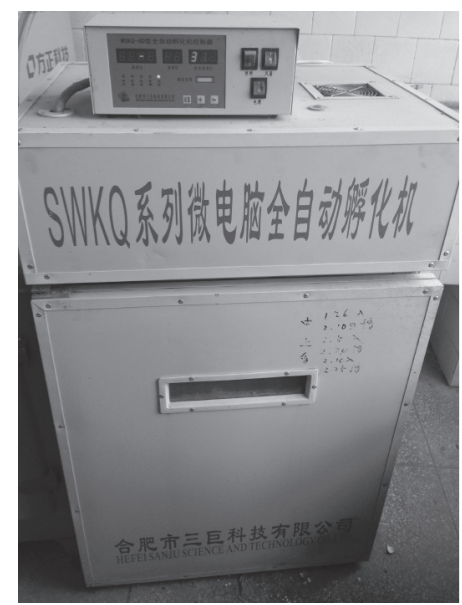

Figure 1 - SWKQ series microcomputer automatic incubator

Incubation temperature was maintained at $37.8^{\circ} \mathrm{C}$ throughout the incubation period, and $60 \%, 50 \%$, and $65-70 \%$ relative humidity were provided during the pre-incubation, middle, and late periods of incubation respectively. The incubator was equipped with electric fans, which continuously ran during incubation to ensure the normal respiratory metabolism of the quail embryos. The egg was automatically turned once every $2 \mathrm{~h}$ to allow uniform heating of the egg.

\section{Determination of the embryo length}

Quail incubation period is $16-17 d$. One egg was randomly selected from the incubator per day and broken to measure embryo length using a vernier caliper. Embryos were photographed to determine their morphological development.

\section{Data analysis}

Quail embryo length was analyzed using the regression model in the statistics software SPSS (2007).

\section{RESULTS}

\section{Morphological development of quail embryos}

The morphological development process of quail embryos is shown in Figure 2. On day 1 of incubation, the embryo begins to grow, and the tracheal primordia appear. On day 2 , blood vessels appear on the surface of the vitellus ovi (yolk), blood appears at center or around the blastoderm. On day 3, the eyeball begins to be pigmented, and the embryo becomes apparent. On day 4 , the eyes have become transparent, and the head is considerably enlarged. On day 5, the eye starts to pigment, and the legs are formed. On day 6, the head and the body are clearly differentiated, the legs become longer, and the wings appear. On day 7, the whole embryo was well defined, and the beak appears. On day 8, fine down appears on the back of the body, and the toes are completely separated from each other. On day 9, the back is longer, and little down and quills also appeared in other parts of the body. On day 10 , fine down appears on the head, and the beak and feet begin to keratinize. On day 11, the entire body is covered with down, and the embryo starts to rotate, turning its head towards the air cell. On day 12, the appearance of the embryo was like body structures are fully developed. On day 13 , the embryo continues to grow, and the volume amniotic fluid starts to decrease, and the air cell becomes inclined. On day 14, the body is elongated. On day 15, the air cell is pierced by the beak, and pulmonary respiration begins. On day 16, the amniotic membrane dropped off; the yolk has been fully absorbed, the eggshell is pipped, and the hatchling leaves the eggshell starts to vocalize. On day 17 , most of the quails had hatched.

\section{Regression analysis of embryo length as a function of incubation day}

Figure 2 clearly shows that the quail embryos grew very fast between days 3 and 9 of incubation, presenting a linear trend. On day 9, quail embryo length reached $2.2 \mathrm{~cm}$ on average. Embryo growth and development were relatively slow on days 10-16 of incubation. Embryo length reached $3.6 \mathrm{~cm}$ on average.

The regression analysis of embryo length as a function of embryo age is shown in Figure 3. The data obtained by the regression curve was coincident with the change in the observed value of the embryo length, as well as the data obtained by linear regression. The data obtained by logarithmic regression and exponential regression were very far from a change in the observed value of the embryo length. Table 1 shows that the highest fitting degree (0.996) was obtained for the curvilinear regression equation. Furthermore, the significance of the regression coefficients $x^{2}$ and $x$ was very high $(p<0.01)$. Therefore, the best regression equation of quail embryo length was a function of embryo age was: $y=-0.464+0.325 x-0.004 x^{2}$, where $y$ is embryo length, and $x$ is embryo age. 


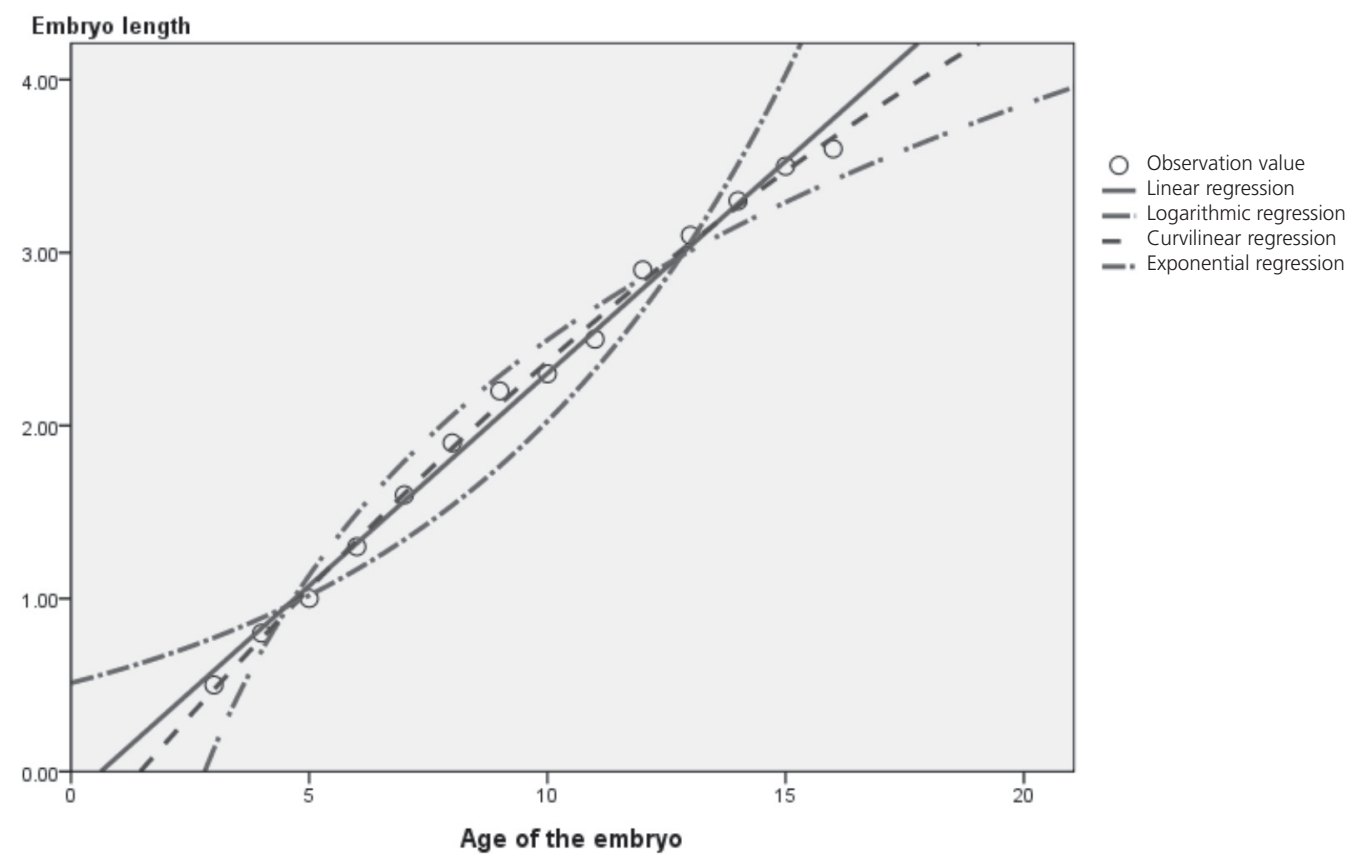

Figure 2 - Regression curves of the length of the quail embryos on the age of the embryo

\section{DISCUSSION}

In this study, the morphological development of quail embryos was studied. On day 6 of incubation, the head and body were clearly differentiated, the legs became longer, and the wings appeared. The trend of embryo length change as a function of age shows that the embryos grew very fast between days 3 and 9 of incubation, after which its growth rate decreased. Embryo length was about $2.2 \mathrm{~cm}$ on day 9 .

The cleavage pattern of quail and duck embryos is discoidal during early embryogenesis (Li et al., 2002), differently from mammals, which present rotary cleavage (Zhang et al., 1994), and from amphibians, which cleavage is holoblastic (Luo, 2007). Hatching ages differ among avian species (16 days in quails, 21 days in chickens, and 28 days in ducks). The early embryonic development state of the various birds varies, as reflected by the differences in the times of tissue and organ formation and differentiation (Luo et al., 2015)

At hatch, stillbirth happened frequently. Early embryonic mortality (1-6d) may be attributed to poor nutrition level and health status of the breeding quails, dietary vitamin $A$ and vitamin $D$ deficiencies, or inadequate egg storage leading to bacterial contamination. The mesentery relaxed, the air chamber flew during egg transportation, and egg storage period longer than two weeks can also cause early mortality of quail embryos. Intermediate embryo mortality (7-14d) results from the incubation of dirty eggs, excessively high incubation temperature, poor of ventilation, among other causes. Late embryo mortality (15-16d) is mainly caused by poor ventilation in the incubator, lack of oxygen, excessive or deficient humidity levels. Also, only fresh eggs (not more than 7-10 days of storage) with the uniform size $(3.5 \mathrm{~cm} \times 2.5 \mathrm{~cm})$ should be incubated. During transportation from the breeder farm to the hatchery, eggs should not be shaken, and before setting, eggs should be disinfected.

\section{REFERENCES}

Chang GB. Study on two wild and domestic quail species evolutionary divergence level [doctoral]. Jiangsu (CN): Yangzhou University; 2003. $158 \mathrm{p}$.

Li BC, Chen GH, Ding JT, et al. The early development of chicken embryo research. Journal of Veterinary Science \& Animal Husbandry 2002;33(3): 230-234.

Lin QL. Quail high-efficiency breeding technology. Nanjin: Jindun Publishing House; p. 14-21

Luo J, Xiao YM, Luo KK, et al. (Andrias davidianus) chinese giant salamander embryo fetal development. The Progress of Natural Science 2007;11:1492-1499.

Luo J, Liu HH, Dai F, Jin HB, Wu QF. Isolation of duck early embryos and morphology observation in differential embryonic phases. China Poultry 2015;37(10):16-20.

Pang YZ. Research and application of the technology of the male and female of quail. Beijing: China Agricultural Press; 2009.

SPSS. SPSS for windows. Version 16.0. Chicago; 2007.

Zhang TY. Lectures on the development of animal embryos (1)-the early development of animal embryos (J). Biology Bulletin 1994;29(7):26-28. 
